\title{
Study on Mix Proportion of Recycled Aggregate Permeable Base Material
}

\author{
Jianguang Xie ${ }^{1, a}$ Daizhou Tong ${ }^{1, b}$, Sicheng Jia ${ }^{1, c}$ \\ ${ }^{1}$ Department of civil engineering, \\ Nanjing University of Aeronautics \& Astronautics,Nanjing,210016,P.R.China \\ a*xiejg@nuaa.edu.cn,b471159098@qq.com, c748236026@qq.com
}

Keywords: Objective void ratio in coarse aggregates; Gradation design; Orthogonal design; Compressive strength; Effective void ratio.

Abstract: Based on a simplified theoretical design method which took the objective void ratio in coarse aggregates (VCAobj) as the index, three different gradation designs with VCAobj of 35\% were proposed in this paper. Through the PFC2D software, both the loading and force transferring were simulated on a micro level of the gradation of aggregate skeleton. During the orthogonal tests for the optimized gradation design, three factors (cement aggregate ratio, water cement ratio and recycled aggregate content) were analyzed to draw the conclusion of their influence on the $28^{\text {th }}$ day's compressive strength and effective void ratio.

\section{Introduction}

At present, the amount of construction wastes has accounted for 30 40\% of the total amount of urban wastes in China. The comprehensive utilization of construction wastes can be an effective way to save natural resources and protect the ecological environment [1]. The application of recycled aggregate to the permeable base can help solve some challenging environmental problems, such as construction waste disposal and huge consumption of natural aggregates. Moreover, it enables natural water to infiltrate into the soil, which will have both air and water permeability. In the meantime, the threats from water damage and ground settlement will be reduced. Based on the application of recycled aggregate to the permeable base, this paper has carried out experimental research on the mix proportion of recycled aggregates.

\section{Gradation design}

\subsection{Study on a simplified theoretical method of gradation based on $\mathrm{VCA}_{\mathrm{obj}}$}

When aggregates with the particle size of $4.75 \sim 26.5 \mathrm{~mm}$ are accumulated in different ratios, the corresponding packing effects are various, which can show different void ratios in coarse aggregates (VCA). Surely there is a maximum void ratio in coarse aggregates ( $\mathrm{VCA}_{\max }$ ) and a minimum void ratio in coarse aggregates $\left(\mathrm{VCA}_{\min }\right)$, and the other VCA values will range between these two values.

Based on the volume design method, a simplified theoretical method of gradation design had been proposed to determine the proportion of coarse aggregates towards the VCAobj in each gradation.

The VCA value in each gradation was measured from the accumulated single particle after vibration molding, as shown in Table 1. Viewing form the results, it can be found that although the particle sizes of each grade are different, there is no significant difference among VCA values in each gradations. So it's reasonable to consider the VCA value of the aggregates with particle size in the range of $4.75 \sim 26.5 \mathrm{~mm}$ as the VCAmax.

Table 1 VCA of aggregates with different particle size grades after the vibration molding

\begin{tabular}{cccccc}
\hline Particle size $(\mathrm{mm})$ & $4.75 \sim 9.5$ & $9.5 \sim 13.2$ & $13.2 \sim 16$ & $16 \sim 19$ & $19 \sim 26.5$ \\
\hline VCA $(\%)$ & 41.0 & 40.7 & 41.5 & 41.2 & 41.5 \\
\hline
\end{tabular}


Based on the theory of maximum dense curve and the theory of interference, the best compact structure can be formed by the step-by-step filling method. It is found that the VCA value decreases to $33.5 \%$ when the mass ratio of five particle sizes in Table 1 is 1: 1.6: 0.72: 0.84: 0.84. It can be concluded that VCAmin can be achieved for the aggregates with the particles sizes in the range of $4.75 \sim 26.5 \mathrm{~mm}$ by using the step-by-step filling method.

The accumulation process of single particle size aggregate is not good for the whole aggregate skeleton to undertake the loading or transfer the force. The optimized embedded accumulation exerts a good mechanical transfer performance. However, if the VCA value is too low, the requirements of

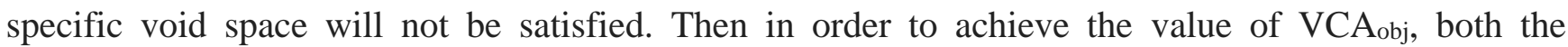
optimized embedded accumulation method and single particle accumulation method should be applied according to a certain proportion. The diagram of gradation skeleton packing is shown in Fig. 1.
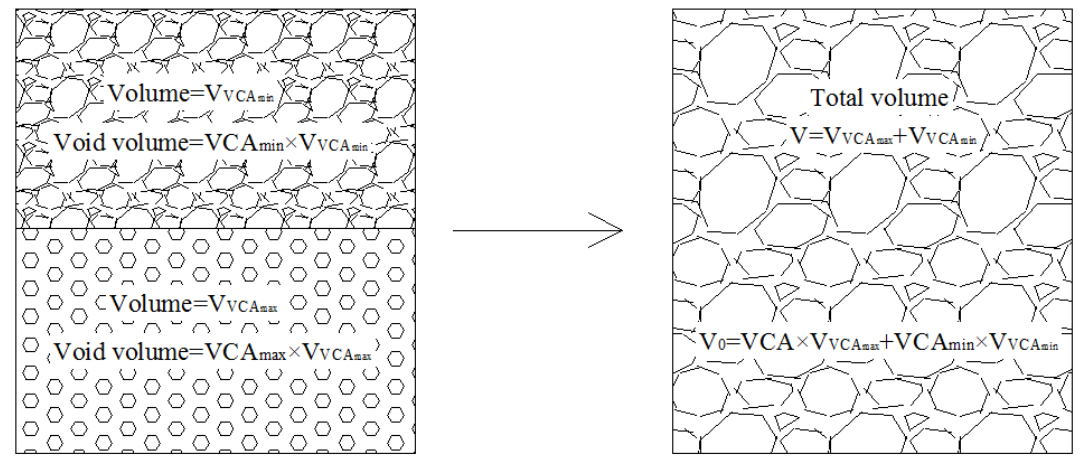

Fig.1 Gradation skeleton packing

Total volume after the combined accumulation is shown in equation 1:

$$
V=V_{V C A_{\max }}+V_{V C A_{\min }}
$$

Where $\mathrm{V}_{\text {VCAmax }}$ means the volume of the specimen portion which gets VCAmax; VvCAmin means the volume of the specimen portion which gets VCAmin.

Total volume $\mathrm{V}_{0}$ of the voids is shown in equation 2:

$$
V_{0}=V C A_{\max } \times V_{V C A_{\max }}+V C A_{\min } \times V_{V C A_{\min }}
$$

The VCA obj values are calculated as equation3:

$$
V C A_{o b j}=V_{0} / V
$$

Then the relationship between the proportion of the volume of single particle accumulation and VCAobj as following:

$$
\frac{V_{V C A_{\min }}}{V}=\frac{V C A_{\max }-V C A_{\mathrm{obj}}}{V C A_{\max }-V C A_{\min }}
$$

With the process of vibration molding for the particles in the range of $4.75 \sim 26.5 \mathrm{~mm}$, the VCAmin value will be 0.335 , while the VCAmax value will be 0.415 . Then, the equation 4 will transfer to the following equation:

$$
\frac{V_{V C A_{\min }}}{V}=\frac{0.415-V C A_{\mathrm{obj}}}{0.08}
$$

According to the above equations, any VCA values can be obtained by controlling the ratio between VvCAmin and VvCAmax. In order to verify the applicability of the simplified theoretical design method which took VCAobj as the index, the VvCAmin and VvCAmax were mixed in different proportions, 
and the actual VCA values were measured, as shown in Fig. 2.

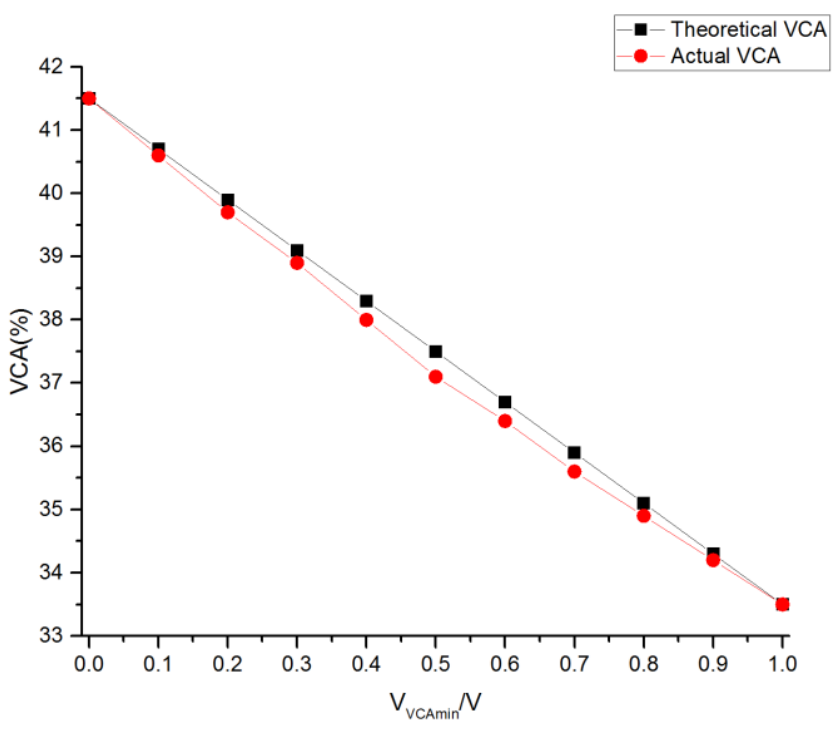

Fig.2 Theoretical and actual values of VCA

Viewing from the results obtained from the simplified theoretical method, it was found that the theoretical value and the actual value depart slightly from each other as shown in Fig. 2. The actual value showes a little bit lower. With the values of $\mathrm{VCA}_{\max }$ and $\mathrm{VCA}_{\text {min }}$ getting closer, the difference between the theoretical and actual value will be even larger. This phenomenon can be explained by the fact that the total volume will decrease after the accumulation process (i.e. $V \leq V_{V C A m i n}+V_{V C A m a x}$ ), and then the actual value of VCA will decrease accordingly.

All in all, the theoretical value is still close to the actual value. Meanwhile, the change rule of $\mathrm{VCA}_{\max }$ and $\mathrm{VCA}_{\text {min }}$ based on different mixing ratio agrees with that obtained from the tests. Then the feasibility of applying the simplified theoretical method to determine the proportion of each grade based on the $\mathrm{VCA}_{\text {obj }}$ can be verified.

\subsection{Gradation design based on the index of $\mathrm{VCA}_{\mathrm{obj}}$}

The gradation design of permeable base materials using recycled aggregate chose VCA $A_{\text {obj }}$ as the

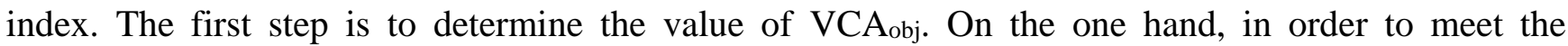
requirement of certain water permeability, the void ratio of material is supposed be $20 \sim 25 \%$. On the other hand, in order to meet the requirement of physical and mechanical properties, the volume of cement paste of the material should be $10 \sim 15 \%$ of the total volume [2-3]. Taking those two aspects into consideration, the VCAobj value was set to $35 \%$. Based on a simplified theoretical design method, three different gradation designs with the VCA value of $35 \%$ were proposed. The particle size of the first gradation was $4.75 \sim 9.5 \mathrm{~mm}$, and the particle size of the third gradation was 19 26.5 mm. Both the first and third gradation were large particle size gradation. The details of these three different gradations are shown in Table 2.

Table 2 Void ratios of target sieve pores from three different gradations

\begin{tabular}{ccccccc}
\hline $\begin{array}{c}\text { Number of } \\
\text { gradation }\end{array}$ & 4.75 & 9.5 & 13.2 & 16 & 19 & 26.5 \\
\hline 1 & 0 & 34 & 60 & 72 & 86 & 100 \\
2 & 0 & 26 & 52 & 64 & 78 & 100 \\
3 & 0 & 17 & 43 & 55 & 69 & 100 \\
\hline
\end{tabular}

\subsection{Microscopic mechanical simulation of gradation skeleton}

The gradation design based on $\mathrm{VCA}_{\text {obj }}$ ensures the gradation skeleton to satisfy the requirement of specific VCA value, while VCA is not the only deciding factor. The gradation skeleton also plays an 
important part in undertaking and transferring the external force. To analyze the property in coarse aggregates, a mechanical simulation of the gradation of aggregate skeleton was carried out in this paper on a micro level by using PFC2D. Then the form of mechanical transmission between particles was analyzed, and the merits and demerits of three different gradation skeletons with the same VCA value were evaluated.

The gradation skeleton is a kind of aggregation of accumulated unbound particles, and the particles are considerably small. Thus, it is reasonable to use discrete element method to set up the linear contact stiffness model. The parameters of the simulated samples [4] are shown in Table 3.

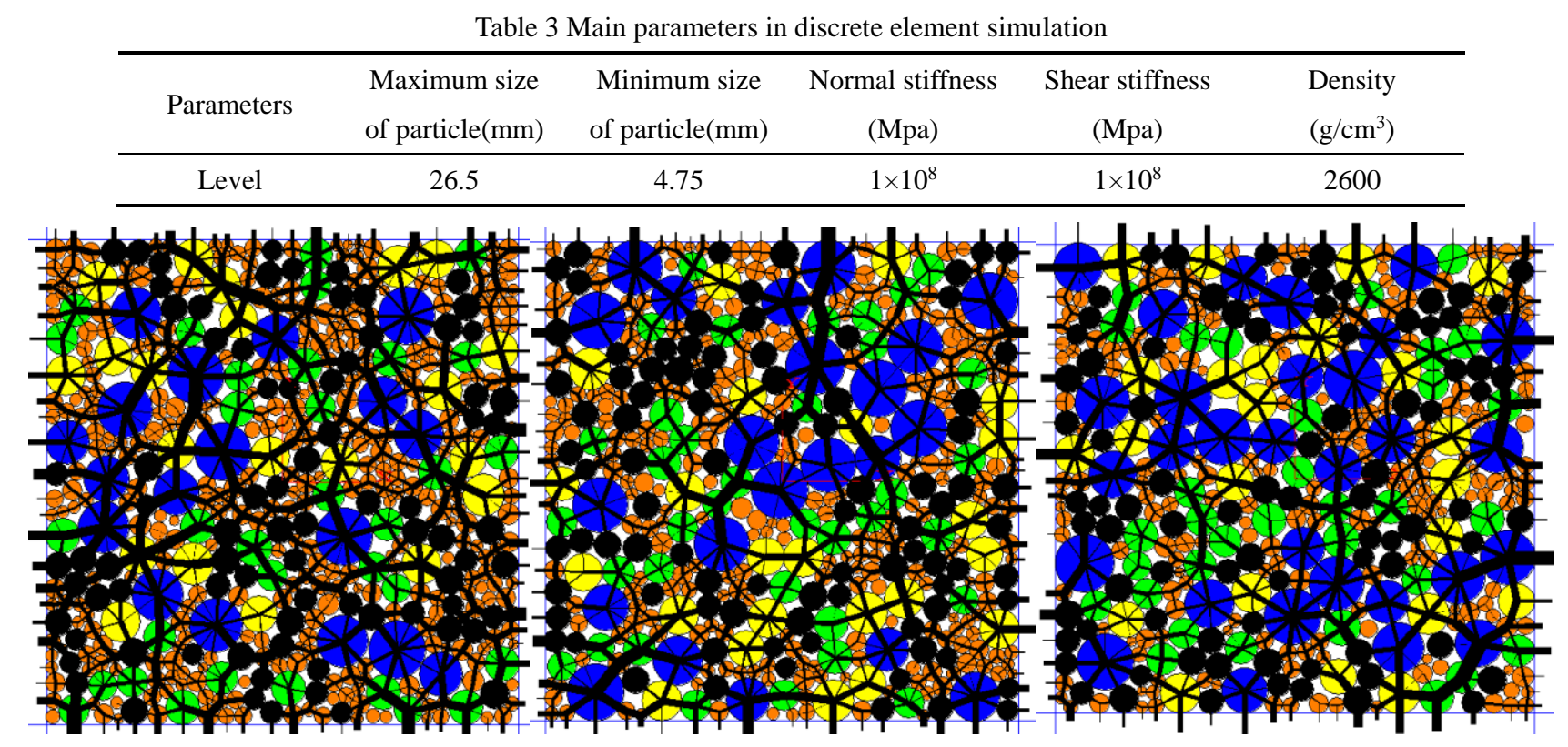

Fig.3 Gradation 1 3 of force chain transfer diagram

The black net line indicates the contact stress between particles or the force between the particle and boundary wall. And the thicker black net line means greater contact stress. In order to evaluate these three gradation skeletons, the simulation of each gradation skeleton was repeated for 10 times, and the maximum contact stress was obtained after all the simulation, as shown in Fig. 4.

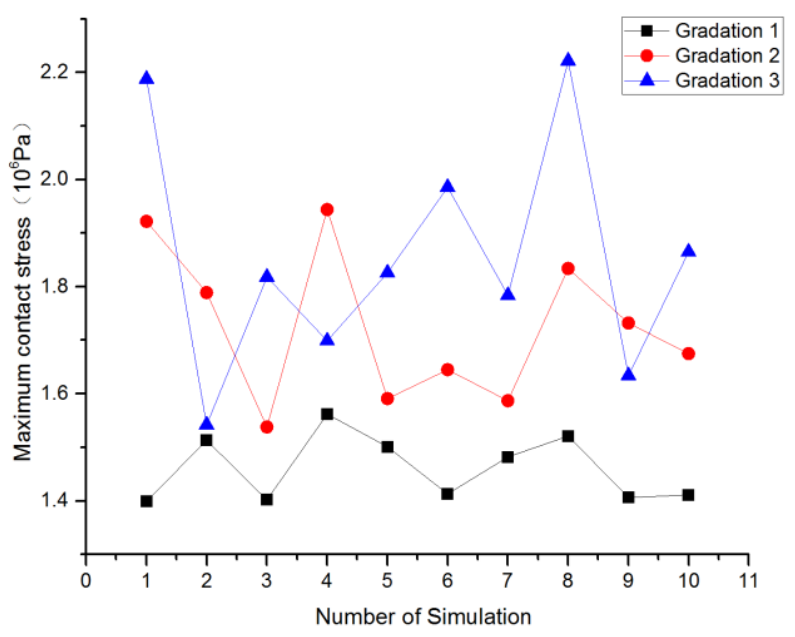

Fig.4 Maximum contact stress of three kinds of gradation

Under the same loading condition, when the maximum contact stress came to bigger in micro mechanics, the strength became smaller in the macro mechanics. The average value and variance of the maximum contact stress of Gradation 1 are the smallest, and those of Gradation 3 are the largest. The simulation results showed that the larger particle size could transfer more contact stress which was more likely to result in the phenomenon of stress concentration and larger discreteness. Gradation 1 
which has smaller particle size showed better skeleton stability and achieves better mechanical property.

\section{Composition design of cement paste}

\subsection{Design on orthogonal tests}

It is of great significance to determine the composition of recycled aggregate permeable base materials. The base usually consists of three contents, including the cement aggregate ratio, the water cement ratio, and the recycled aggregate content. After four levels were allocated to each factor, orthogonal tests could be carried out. The experimental gradation set the VCA value as 35\% and took the compressive strength at the $28^{\text {th }}$ day and the effective void ratio as two indexes of the orthogonal tests.

Taking $28^{\text {th }}$ day's compressive strength and effective void ratio as the indexes of the orthogonal test, at the end of the standard maintenance of specimens, the $28^{\text {th }}$ day's unconfined compressive strength was measured by the press machine, and the effective void ratio of the specimens was measured by the hydrostatical balance.

\begin{tabular}{cccccc}
\multicolumn{7}{c}{ Table 4 Results of the orthogonal test } \\
\hline Number & A & B & C & $\begin{array}{r}28^{\text {th }} \text { day's compressive strength } \\
(\text { Mpa })\end{array}$ & $\begin{array}{c}\text { Effective porosity } \\
(\%)\end{array}$ \\
\hline $1-1$ & $1(1: 9)$ & $1(0.40)$ & $1(25 \%)$ & 5.78 & 19.7 \\
$1-2$ & 1 & $2(0.45)$ & $2(50 \%)$ & 5.82 & 20.2 \\
$1-3$ & 1 & $3(0.50)$ & $3(75 \%)$ & 6.01 & 20.6 \\
$1-4$ & 1 & $4(0.55)$ & $4(100 \%)$ & 5.94 & 21.0 \\
$2-1$ & $2(1: 10)$ & 1 & 2 & 3.11 & 21.5 \\
$2-2$ & 2 & 2 & 1 & 5.74 & 20.8 \\
$2-3$ & 2 & 3 & 4 & 4.63 & 21.8 \\
$2-4$ & 2 & 4 & 3 & 5.62 & 21.3 \\
$3-1$ & $3(1: 11)$ & 1 & 3 & 2.76 & 23.2 \\
$3-2$ & 3 & 2 & 4 & 3.67 & 23.6 \\
$3-3$ & 3 & 3 & 1 & 4.71 & 22.8 \\
$3-4$ & 3 & 4 & 2 & 4.69 & 23.2 \\
$4-1$ & $4(1: 12)$ & 1 & 4 & 1.96 & 24.9 \\
$4-2$ & 4 & 2 & 3 & 3.23 & 24.8 \\
$4-3$ & 4 & 3 & 2 & 4.78 & 24.3 \\
$4-4$ & 4 & 4 & 1 & 3.83 & 24.5 \\
\hline
\end{tabular}

\subsection{Analysis of the orthogonal test}

The range $R$ is related with the influence degree of the factors. Larger range means that the factor has more obvious effect on the indexes in the test. $R$ is the difference between the maximum and the minimum:

$$
R=\left|k_{\max }-k_{\min }\right|
$$

Table 5 shows the effect of the factors on the indexes. 
Table 5 Visual analysis results of orthogonal test

\begin{tabular}{ccccccc}
\hline Index & Factor & $\mathrm{K}_{1}$ & $\mathrm{~K}_{2}$ & $\mathrm{~K}_{3}$ & $\mathrm{~K}_{3}$ & $\mathrm{R}$ \\
\hline 28 ${ }^{\text {th }}$ day' & $\mathrm{A}$ & 5.89 & 4.78 & 3.96 & 3.45 & 2.44 \\
compressive & $\mathrm{B}$ & 3.40 & 4.62 & 5.03 & 5.02 & 1.63 \\
strength & $\mathrm{C}$ & 5.02 & 4.60 & 4.41 & 4.05 & 0.97 \\
\hline Effective & $\mathrm{A}$ & 20.38 & 21.35 & 23.20 & 24.63 & 4.25 \\
porosity & $\mathrm{B}$ & 22.33 & 22.35 & 22.38 & 22.50 & 0.17 \\
& $\mathrm{C}$ & 21.95 & 22.30 & 22.48 & 22.83 & 0.88 \\
\hline
\end{tabular}

i ) Comparison among the effect of each factors on the indexes

The results of table 4 and 5 can be used to analyze the effects of various factors on the indexes. According to the influence on the $28^{\text {th }}$ day's compressive strength, the factors were in the order of A (cement aggregate ratio) $>\mathrm{B}$ (water cement ratio) $>\mathrm{C}$ (recycled aggregate ratio). As for the influence on the effective void ratio, the range becomes: A (cement aggregate ratio) $>\mathrm{C}$ (recycled aggregates ratio) $>\mathrm{B}$ (water cement ratio).

ii) Change rule between factor levels and index values

The actual value of each factor level was marked in the horizontal coordinate, and the corresponding index values were marked in the vertical coordinate. Then the change rule between factor levels and index values could be obtained, as shown in Fig. 5.
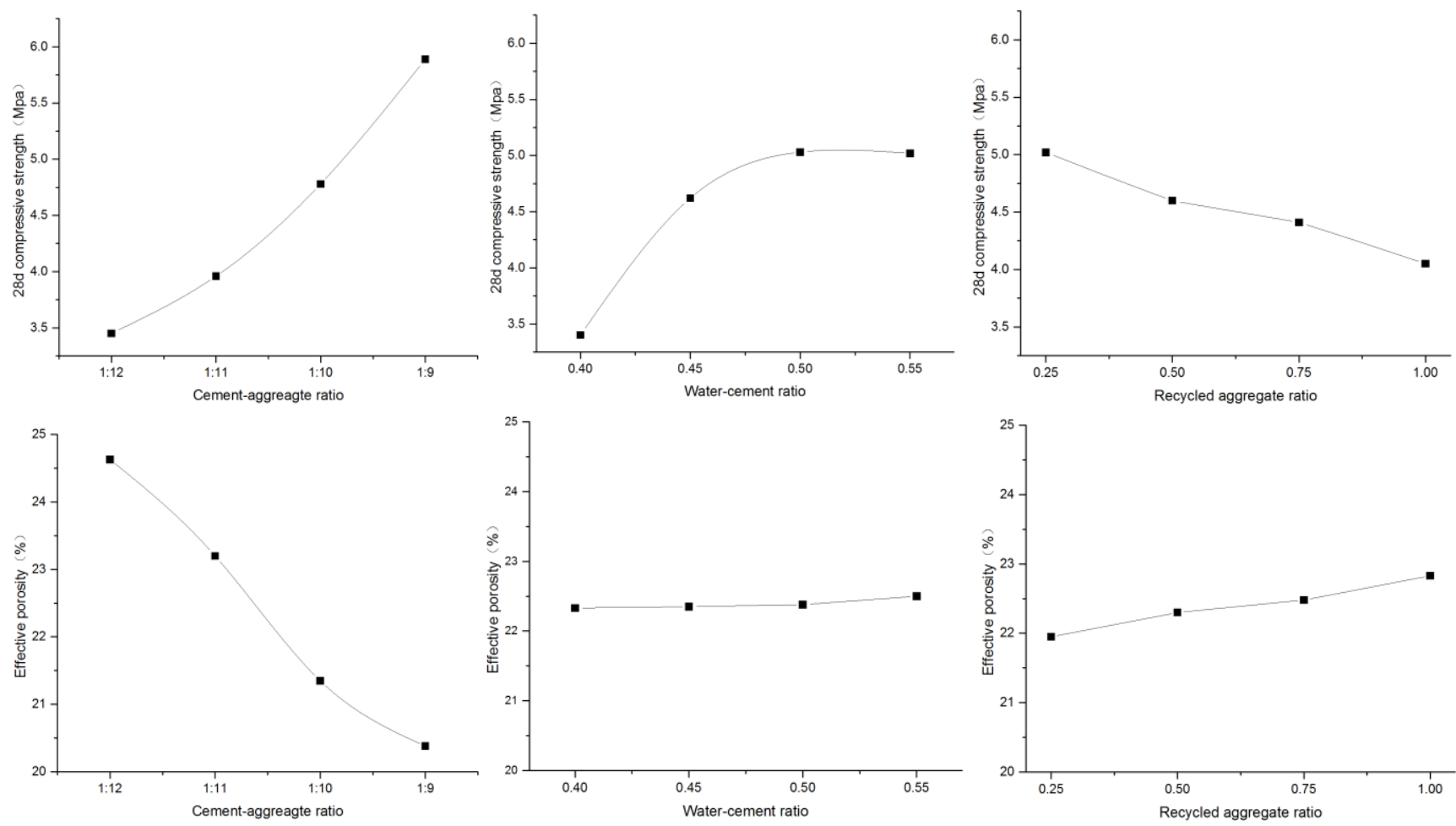

Fig.5 Effects of factors on Indexes

With the increase of cement content, the compressive strength increased, and the effective void ratio decreases gradually. According to the requirement of practical engineering, the cement aggregate ratio was controlled in the mix design to ensure the performance of recycled aggregates. The mixing amount of recycled aggregates and water cement ratio had no obvious effects on the indexes, which 
was mainly because the interaction between two factors led to the difficulty of finding relationship between indexes and the change of single factor level. So it needs further analysis to find out the interrelation between the recycled aggregates and water.

iii) Interrelation between the recycled aggregates and water.

For the reason that recycled aggregate was highly water-absorbing, some water was absorbed into the voids of aggregates, leading to the consequence that some water cannot be combined with cement, and either be used as the free water in the cement paste to promote hydration. The water consumption was closely related to the amount of recycled aggregates, so the results of factor B (water cement ratio) and factor $\mathrm{C}$ (recycled aggregate ratio) in the orthogonal test need to be analyzed respectively.

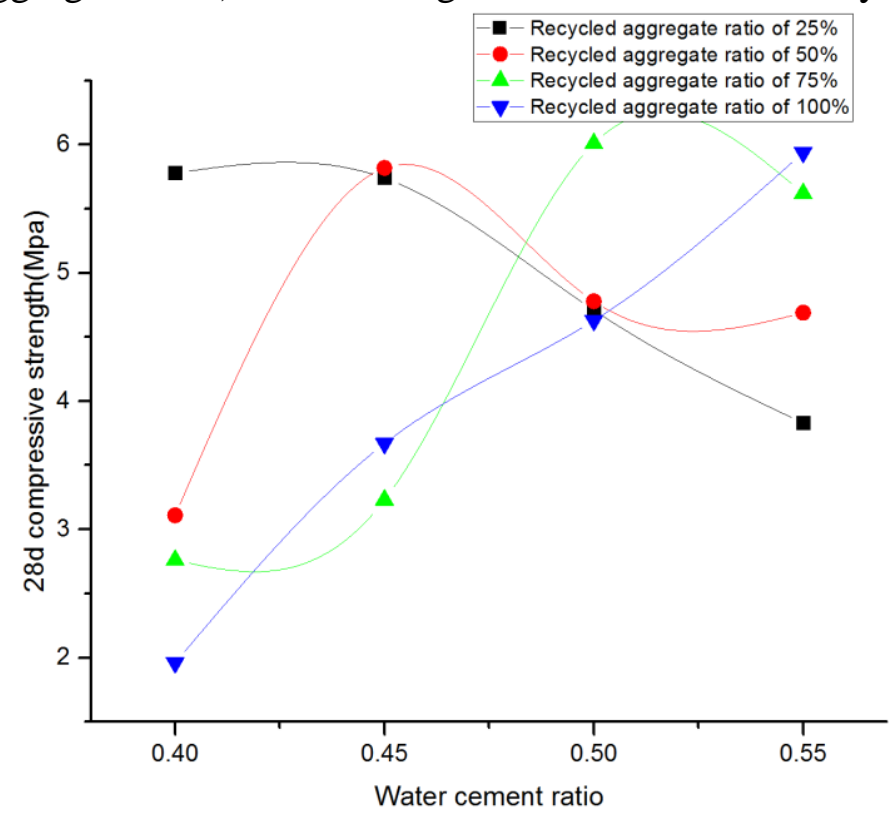

Fig.6 The relationship of water cement ratio and strength with different amount of recycled aggregates

As seen in Fig.6, with the increase of water cement ratio, the $28^{\text {th }}$ day's compressive strength increased and then decreased from the peak. It could be concluded that the water cement ratio optimum water ratio corresponding to the peak strength meant the optimal water cement ratio, which was $0.40 \sim 0.45,0.45 \sim 0.50,0.50 \sim 0.55$ and above 0.55 when recycled aggregate ratio is $25 \%, 50 \%, 75 \%$ and $100 \%$, respectively.

The optimum water cement ratio for permeable base using natural aggregates was 0.39 0.41[5], which contained the water content needed for hydration effect and hydration promoting. As for the permeable base with recycled aggregate, high water absorption made the water consumption include free water in the mixing process of recycled aggregates. Fig. 6 showed that when the recycled aggregate content increased by $25 \%$, the water cement ratio increased about 0.5 . And the amount of absorbent free water in the mixing process of recycled aggregates could be calculated as $70 \%$ of water absorbed by recycled aggregates. So the water required for recycled aggregates in the mixing can be expressed as:

$$
W=0.4 C+0.7 w A
$$

Where $\mathrm{W}$ means water consumption for mixing; w refers to the water absorption of aggregates $(\%)$; $\mathrm{C}$ refers to the amount of cement $(\mathrm{g}) ; \mathrm{M}$ means the mass of the aggregates $(\mathrm{g})$.

\section{Conclusions}

This paper studied the mix proportion of recycled aggregate permeable base material through the design of gradation and cement paste. Based on the results and analysis, the following conclusions 
were made:

1. Based on the volume method, a simplified theoretical method of gradation design was proposed with the index of $\mathrm{VCA}_{\mathrm{obj}}$. This method added a certain proportion of single particle aggregate to the optimized embedded skeleton structure of accumulated coarse aggregates to get VCAobj. Then the relationship of the proportion of single particle and the VCAobj can be derived accordingly.

2. Based on the simplified theoretical method, three different gradation designs with the VCA $\mathrm{obj}$ value of $35 \%$ were designed. With the help of PFC2D software, both the loading and force transferring were simulated and analyzed on a micro level of those three gradation skeletons. According to the results of simulation, it was found that the gradation 1 had relatively smaller particles and better skeleton stability and mechanical properties.

3. Three factors, including the cement aggregate ratio, the water cement ratio, and the recycled aggregate content, were analyzed to find the rules of their influence on the composition of the cement paste. And the theoretical formula of the actual water consumption of the material of the recycled aggregate permeable base can be obtained.

\section{Acknowledgments}

This work was supported by National Science and Technology Support Program(2015BAL02B00) and Science and Technology Program Project in Jiangsu Province（SBE2015310591)

\section{References}

[1] D. Liu. Research on the Present Situation and Countermeasures of Construction Waste Management in China. Management and Technology of Small and Medium Sized Enterprises. 5(2010) 121-122.

[2] Schaus L K. Porous Asphalt Pavement Designs. University of Waterloo, 2007.

[3] Ferguson B K, Porous Pavement. CRC Press LLC, 2005.

[4] S. Tu, Structure and Performance of High-Performance Graded Crushed-Stone Base Course Based on the Interaction. Chang'an University, 2013.

[5] Q. Zhang, J. Chen, B, Tie. Study on Mix Proportion Design Method of Lean Concrete Permeable Base Material. Journal of Highway and Transportation Research and Development. 23(6) (2006) 56-59. 